\title{
Cytokines and cellular interactions in inflammatory synovitis
}

\begin{abstract}
William P. Arend
Department of Medicine, Division of Rheumatology, University of Colorado Health Sciences Center B115, 4200 East Ninth Avenue, Denver, Colorado 80262, USA. Phone: (303) 315-6666; Fax: (303) 315-5540; E-mail: william.arend@uchsc.edu.
\end{abstract}

Considerable evidence accumulated over the past decade has clarified some of the mechanisms of cell-cell interactions in rheumatoid synovitis and the important roles of IL- 1 and TNF- $\alpha$ in inflammation and tissue destruction $(1,2)$. These advances in knowledge have led to the development of novel anticytokine therapies for rheumatoid arthritis (RA). However, in spite of these successes, disagreement remains concerning the relative roles of $\mathrm{T}$ cell-dependent versus $T$ cell-independent mechanisms and of IL- 1 versus TNF- $\alpha$ in the initiation and perpetuation of rheumatoid synovitis.

Studies in experimental animal models of inflammatory arthritis have shed some light on these issues in RA, although it is generally recognized that no animal model is completely equivalent to the human disease. The article by Niki et al. in this issue of the JCI provides some interesting new findings on effects of IL-1 on synovitis (3) and speaks to some of the broader questions regarding the cells and cytokines that drive this disease.

\section{Arthritis in IL-1 $\alpha$ transgenic mice}

Niki et al. (3) describe the spontaneous development in IL- $1 \alpha$ transgenic mice of inflammatory arthritis with a predominance of neutrophils and macrophages in the diseased tissue. Patterns of transgene expression may complicate the interpretation of their findings somewhat. The authors report that five of the seven founders (F0) they generated did not develop arthritis and, indeed, these animals expressed a minimum level of hIL- $1 \alpha$ mRNA or protein. However, in the two F0 mice that developed arthritis, many cell types exhibited hIL- $1 \alpha$ mRNA expression, but the protein was produced predominantly by macrophages and fibroblast-like synoviocytes. Thus, the nature of integration of the transgene and the relative cell-type specificity of translation may have influenced the resulting phenotype.

The data of Niki et al. (3) suggest that the bone marrow in hIL- $1 \alpha$ transgenic mice produces increased numbers of neutrophils and macrophages, with mature cells found particularly in the joints. Consistent with the known stimulatory effects of IL-1 on GM-CSF production, the authors also observed increased GM-CSF levels in the serum and in supernatants of cultured bone marrow and synovial cell. The effects of GM-CSF on arthritis in this model may have been mediated not only through enhancing hematopoiesis, but also through the stimulatory effects of this cytokine on peripheral neutrophils and monocytes (4). In mice, both collageninduced arthritis (CIA) and IL1-enhanced, antigen-induced arthritis are dependent upon GM-CSF, with effects probably mediated both in the bone marrow and peripherally $(4,5)$. Confirmation that the same is true in the hIL- $1 \alpha$ transgenic mice must await further studies on cytokine and chemokine levels in arthritic joints and on the role of membrane-bound IL- $1 \alpha$ on synovial cells.

This work shows that inflammatory arthritis in animals may develop in a $\mathrm{T}$ cell-independent manner and that antigen-presentation to $T$ cells is not necessarily required. The role of $T$ cells in RA remains controversial, and it seems likely that both $\mathrm{T}$ cell-dependent and -independent mechanisms are present to variable degrees, both over time in an individual patient and between patients (6). T-cell induction of antigen-specific immune responses is generally considered to be important in the pathophysiology of RA, although abnormalities in T-cell homeostasis may also be involved (7). Contracted T-cell diversity and the emergence of large clonal T-cell populations in RA patients may contribute to the development of autoimmune responses (8). However, stimulation of the innate immune system may not only shape adaptive immune responses, but may also lead directly to cytokine release from dendritic cells and macrophages and thus to T cell-independent inflammation and tissue destruction (9).
TNF- $\alpha$, IL-1, and IL-1Ra in inflammatory synovitis

The importance of IL- 1 and TNF- $\alpha$ in experimental animal models of inflammatory arthritis and in RA has been extensively studied and reviewed (10). These cytokines both enhance the migration of cells into the joint and stimulate matrix metalloproteinase production in synovial fibroblasts and chondrocytes. Analysis of the effects of therapeutic TNF- $\alpha$ blockade has highlighted the importance for the successful treatment of RA of inhibiting cell migration (10). In the current study, Niki et al. detected high levels of TNF- $\alpha$ mRNA expression in the hIL- $1 \alpha$ transgenic mice, consistent with the known stimulatory effects of IL- 1 on TNF- $\alpha$ production (3). Conversely, TNF- $\alpha$ is also well known to stimulate IL-1 production.

IL-1 also plays a key role in rheumatoid synovitis, both in experimental animal models and in RA. Intra-articular injection of IL- $1 \alpha$ or of IL- $1 \beta$ or local production of IL- $1 \beta$ after gene transfer to the rabbit joint can induce an inflammatory arthritis with pathological changes closely resembling those seen in RA $(11,12)$. Whereas inhibition of TNF- $\alpha$ in CIA is primarily anti-inflammatory, IL-1 blockade more potently prevents destruction of cartilage and bone in this model (13). In humans, although the expression of these inflammatory mediators varies between patients, macrophages containing IL-1 predominate at the cartilage-pannus junction in early RA, and TNF- $\alpha$-containing cells are relatively scarce (14). Moreover, recent studies on RA synovia, which used immunohistochemistry to detect cytokine-producing rather than cytokine-binding cells, demonstrated prominent staining for IL-1 $\beta$ in macrophages and fibroblasts and for IL- $1 \alpha$ in vascular endothelial cells, but relatively sparse staining for TNF- $\alpha$ (15). The clinical efficacy of anti-TNF- $\alpha$ therapies in RA, observed in only a subset of 
patients, may be related to the relative ease of inhibition due to the low and variable levels of TNF- $\alpha$ present. However, RA appears to be different from CIA, as TNF- $\alpha$ blockade in the human disease clearly prevents destruction of cartilage and bone $(16,17)$.

An imbalance between IL-1 and its naturally occurring inhibitor IL-1Ra may also predispose to inflammatory arthritis (18). Thus, the incidence and severity of CIA are significantly reduced in IL-1Ra transgenic mice and increased in IL-1Ra knockout mice (19). The spontaneous RA-like disease seen in IL-1Ra knockout mice bred on the $\mathrm{BALB} / \mathrm{cA}$ background is accompanied by an overexpression of IL-1 $\beta$, IL- 6 , and TNF- $\alpha$ in the joints (20). Since IL- 1 can induce synthesis of all of these cytokines, it appears that physiological levels of IL-1Ra may regulate their excess production. It would be interesting to know the levels of IL-1Ra in the joints of the hIL- $1 \alpha$ transgenic mice in the studies by Niki et al., since IL-1 also can induce production of IL-1Ra (3). The presence of a great excess of IL-1, either membrane-bound or secreted, over IL-1Ra in the synovium of these mice would further support the concept that an imbalance in this system predisposes to disease.

\section{Conclusions}

The observations that IL- 1 and TNF- $\alpha$ are important mediators of inflammation and tissue destruction in RA have led to the development of successful treatments for this disease. However, many questions remain unanswered. How does this disease process begin and what transforms a relatively mild synovitis into a chronic, destructive lesion? What mechanisms are responsible for stimulating IL- 1 and TNF- $\alpha$ production in the rheumatoid synovium, and why is the relative level of production of IL-1Ra by synovial macrophages inadequate? What is the importance of other cytokines in RA such as IL-6, IL-8, IL-12, IL-15, IL-17, and IL-18, and will inhibition of these cytokines either alone or in combination with blockade of IL- 1 or TNF- $\alpha$ offer greater therapeutic efficacy and safety? A greater understanding of the contributions of the innate and adaptive immune systems in RA may lead to the development of more specific and effective therapeutic approaches.

1. Arend, W.P., and Dayer, J.-M. 1995. Inhibition of the production and effects of interleukin-1 and tumor necrosis factor $\alpha$ in rheumatoid arthritis. Arthritis Rheum. 38:151-160.

2. Feldmann, M., Brennan, F.M., and Maini, R.N 1996. Role of cytokines in rheumatoid arthritis Annu. Rev. Immunol. 14:397-440.

3. Niki, Y., et al. 2001. Macrophage- and neutrophildominant arthritis in human IL- $1 \alpha$ transgenic mice. J. Clin. Invest. 107:1127-1135.

4. Campbell, I.K., et al. 1998. Protection from collagen-induced arthritis in granulocyte-macrophage colony-stimulating factor-deficient mice. J. Immunol. 161:3639-3644.

5. Yang, Y.H., and Hamilton, J.A. 2001. Dependence of interleukin-1-induced arthritis on granulocyte-macrophage colony-stimulating factor. Arthritis Rheum. 44:111-119.

6. Fox, D.A. 1997. The role of $\mathrm{T}$ cells in the immunopathogenesis of rheumatoid arthritis. New perspectives. Arthritis Rheum. 40:598-609.

7. Weyand, C.M. 2000. New insights into the patho- genesis of rheumatoid arthritis. Rheumatology. 39(Suppl.):3-8.

8. Koetz, K., et al. 2000. T cell homeostasis in patients with rheumatoid arthritis. Proc. Natl. Acad. Sci. USA. 97:9203-9208.

9. Arend, W.P. 2001. The innate immune system in rheumatoid arthritis. Arthritis Rheum. In press.

10. Feldmann, M., Elliott, M.J., Woody, J.N., and Maini, R.N. 1997. Anti-tumor necrosis factor- $\alpha$ therapy of rheumatoid arthritis. Adv. Immunol. 64:283-350.

11. van de Loo, A.A.J., and van den Berg, W.B. 1990 Effects of murine recombinant interleukin 1 on synovial joints in mice: measurement of patellar cartilage and joint inflammation. Ann. Rheum. Dis. 49:238-245.

12. Ghivizzani, S.C., et al. 1997. Constitutive intraarticular expression of human IL- $1 \beta$ following gene transfer to rabbit synovium produces all major pathologies of human rheumatoid arthritis. J. Immunol. 159:3604-3612.

13. Joosten, L.A.B., et al. 1999. IL- $1 \alpha \beta$ blockade prevents cartilage and bone destruction in murine type II collagen-induced arthritis, whereas TNF$\alpha$ blockade only ameliorates joint inflammation. J. Immunol. 163:5049-5055.

14. Tak, P.P., and Bresnihan, B. 2000. The pathogenesis and prevention of joint damage in rheumatoid arthritis. Arthritis Rheum. 43:2619-2633.

15. Ulfgren, A.-K., et al. 2000. Interindividual and intra-articular variation of proinflammatory cytokines in patients with rheumatoid arthritis: potential implications for treatment. Ann. Rheum Dis. 59:439-447.

16. Bathon, J.M., et al. 2000. A comparison of etanercept and methotrexate in patients with early rheumatoid arthritis. $N$. Engl. J. Med. 343:1586-1593.

17. Lipsky, P.E., et al. 2000. Infliximab and methotrexate in the treatment of rheumatoid arthritis. N. Engl. J. Med. 343:1594-1602.

18. Ma, Y., et al. 1998. Altered susceptibility to collagen-induced arthritis in transgenic mice with aberrant expression of interleukin-1 receptor antagonist. Arthritis Rheum. 41:1798-1805.

19. Arend, W.P. 2001. Cytokine imbalance in the pathogenesis of rheumatoid arthritis: the role of IL-1Ra. Semin. Arthritis Rheum. In press.

20. Horai, R., et al. 2000. Development of chronic inflammatory arthropathy resembling rheumatoid arthritis in interleukin-1 antagonist-deficient mice. J. Exp. Med. 191:313-320. 